\title{
Adolescent male chimpanzees (Pan troglodytes) form social bonds with their brothers and others during the transition to adulthood
}

\author{
Aaron A. Sandel ${ }^{1,2 *}$ (]) $\mid$ Kevin E. Langergraber ${ }^{3,4}$ | John C. Mitani ${ }^{2}$ (])
}

${ }^{1}$ Department of Anthropology, University of Texas, Austin, Texas

${ }^{2}$ Department of Anthropology, University of Michigan, Ann Arbor, Michigan

${ }^{3}$ School of Human Evolution and Social Change, Arizona State University, Tempe, Arizona

${ }^{4}$ Institute of Human Origins, Arizona State University, Tempe, Arizona

\section{Correspondence}

Aaron A. Sandel, Department of Anthropology, University of Texas at Austin, 2201 Speedway, WCP 4.102, Mailcode C3200, Austin,

TX 78712.

Email: aaron.sandel@austin.utexas.edu

\section{Funding information}

Horace H. Rackham School of Graduate Studies, University of Michigan; Leakey Foundation; National Institutes of Health, Grant/Award Number: RO1AG049395; National Science Foundation, Directorate for Social, Behavioral and Economic Sciences, Grant/Award Numbers: 1540259, F031543; Nacey-Maggioncalda Foundation

\begin{abstract}
Social relationships play an important role in animal behavior. Bonds with kin provide indirect fitness benefits, and those with nonkin may furnish direct benefits. Adult male chimpanzees (Pan troglodytes) exhibit social bonds with maternal brothers as well as unrelated adult males, facilitating cooperative behavior, but it is unclear when these bonds develop. Prior studies suggest that social bonds emerge during adolescence. Alternatively, bonds may develop during adulthood when male chimpanzees can gain fitness benefits through alliances used to compete for dominance status. To investigate these possibilities and to determine who formed bonds, we studied the social relationships of adolescent and young adult male chimpanzees $(N=18)$ at Ngogo in Kibale National Park, Uganda. Adolescent male chimpanzees displayed social bonds with other males, and they did so as often as did young adult males. Adolescent and young adult males frequently joined subgroups with old males. They spent time in proximity to and grooming with old males, although they also did so with their age peers. Controlling for age and age difference, males formed strong association and proximity relationships with their maternal brothers and grooming relationships with their fathers. Grooming bonds between chimpanzee fathers and their adolescent and young adult sons have not been documented before and are unexpected because female chimpanzees mate with multiple males. How fathers recognize their sons and vice versa remains unclear but may be due to familiarity created by relationships earlier in development.
\end{abstract}

KEYWORDS

development, kinship, paternal relationships, social relationships

\section{1 | INTRODUCTION}

Strong social relationships or "social bonds" influence the lives of mammals in important ways, and this is especially true for primates (Massen, Sterck, \& de Vos, 2010; Seyfarth \& Cheney, 2012). Given the prevalence of female philopatry and male dispersal in primates,

${ }^{*}$ Author is no longer affiliated with the University of Michigan. prior research has focused on bonds between natal females that are often kin (Silk, 2009). In baboons, females with social bonds often have higher infant survivorship, live longer, and display lower stress levels than those with weak ties (e.g., chacma baboons, Papio ursinus: Crockford, Wittig, Whitten, Seyfarth, \& Cheney, 2008; yellow baboons, Papio cynocephalus: Silk, Alberts, \& Altmann, 2003). Adult male primates also obtain fitness benefits by forging social bonds, including with non-kin when males are the dispersing sex (e.g., male Assamese macaques, Macaca assamensis: Schülke, Bhagavatula, 
Vigilant, \& Ostner, 2010; Young, Majolo, Heistermann, Schülke, \& Ostner, 2014). Notably, male chimpanzees (Pan troglodytes) remain in their natal communities (Goodall, 1986; Nishida, 1979), and as adults form bonds with both maternal half-brothers and nonrelatives, especially males similar in age and rank (Langergraber, Mitani, \& Vigilant, 2007; Mitani, 2009; Mitani, Watts, Pepper, \& Merriwether, 2002). Strongly bonded adult male chimpanzees cooperate to attain high dominance rank (Nishida, 1983; Nishida \& Hosaka, 1996), which is positively related to reproduction (e.g., Boesch, Kohou, Néné, \& Vigilant, 2006; Wroblewski et al., 2009). Affiliative relationships also mediate participation in territorial defense, hunting, food sharing (Mitani \& Watts, 2001; Samuni et al., 2018; Watts \& Mitani, 2001), and may buffer individuals against stress (Wittig et al., 2016).

Despite the importance of social bonds for the lives of many adult primates, the development of such relationships is poorly understood (Alberts, 2019; Amici, Kulik, Langos, \& Widdig, 2019). This is true for some of the most well-studied species where social bonds have been the focus of considerable research, such as chimpanzees (e.g., Pusey, 1990). Although social bonds play a prominent role in the lives of adult male chimpanzees, whether such relationships arise before adulthood remains unclear (Kawanaka, 1989; Pusey, 1990).

Two possibilities exist for the development of social bonds in male chimpanzees. One is that bonds develop during adulthood when male chimpanzees forge social bonds with relatives and peers to help in the acquisition and maintenance of dominance rank (Nishida, 1983; Riss \& Goodall, 1977). Adolescent male chimpanzees, however, do not form decided dominance relationships with their peers (Sandel, Reddy, \& Mitani, 2017). As a consequence, male chimpanzees may wait to establish bonds with others until adulthood, when they begin competing for status. In addition, adolescent males remain relatively asocial, spending considerable time alone and at the periphery of social gatherings (Kawanaka, 1993; Pusey, 1990). As a consequence, it may be difficult for them to forge social bonds until they become more gregarious during adulthood.

A second possibility is that social bonds emerge before adulthood, specifically during adolescence when male chimpanzees become fully independent of their mothers and start to integrate into the adult social world. During adolescence, male chimpanzees spend an increasing amount of time with adult males but remain socially peripheral, as they are frequent targets of aggression and rarely groomed (Kawanaka, 1989; Pusey, 1990). To negotiate the difficult transition to adulthood adolescents may form social bonds with other males (Nishida, 2012; Pusey, 1990). Prior observations of four adolescent males at Gombe National Park, Tanzania, indicated that one preferentially traveled with an older brother, another did so with the alpha male, and a third did so with four unrelated males, including three old males (Pusey, 1990). Similarly, three of seven adolescent male chimpanzees at Mahale Mountains National Park, Tanzania, spent considerable time in close spatial proximity to specific males (Kawanaka, 1989). These observations suggest that adolescent males exhibit social bonds, but more data are necessary to confirm that this actually occurs.

If adolescent male chimpanzees do form social bonds, further research is required to determine who they target as partners.
Qualitative observations indicate that adolescent males seek the company of high-ranking and older males (Nishida, 2012; Pusey, 1990). Recent studies of immature chimpanzees and other primates also suggest that fathers and offspring bias certain affiliative behaviors toward each other (chimpanzees, Pan troglodytes: Lehmann, Fickenscher, \& Boesch, 2006; Murray, Stanton, Lonsdorf, Wroblewski, \& Pusey, 2016; yellow baboons, Papio cynocephalus: Charpentier, Van Horn, Altmann, \& Alberts, 2008; chacma baboon, Papio ursinus: Huchard et al., 2013; mandrills, Mandrillus sphinx: Charpentier, Peignot, Hossaert-McKey, \& Wickings, 2007; rhesus macaques, Macaca mulatta: Langos, Kulik, Mundry, \& Widdig, 2013; Pfefferle, Kazem, Brockhausen, Ruiz-Lambides, \& Widdig, 2014; capuchin monkeys, Cebus capucinus: Godoy, Vigilant, \& Perry, 2016). Research on mature male chimpanzees suggests that bonds preferentially exist between males who are similar in age and between maternal brothers, but not between paternal brothers (Langergraber et al., 2007; Mitani, 2009; Mitani et al., 2002). How age similarity and relatedness influence bond formation in adolescent chimpanzees is unknown.

We investigated the development of male chimpanzee social relationships during the transition to adulthood. To do so, we studied a relatively large cohort of young male chimpanzees at Ngogo in Kibale National Park, Uganda. We observed 10 adolescent chimpanzees ranging in age from 12 to 16 years, comparing their patterns of affiliation to those of eight young adult males ranging in age from 17 to 21 years. We addressed two questions. First, do adolescent male chimpanzees exhibit strong social bonds with other males or do bonds develop later after males reach adulthood? Second, if adolescent males do exhibit social bonds, with whom are they formed?

\section{2 | METHODS}

\section{1 | Ethics statement}

Research in Uganda was approved by the Uganda National Council for Science and Technology. Approval for research in Kibale National Park was granted by the Uganda Wildlife Authority. Research was reviewed by the University Committee on Use and Care of Animals at the University of Michigan and was granted an exemption because animal use was limited to noninvasive behavioral observations.

\section{2 | Study site and subjects}

Sandel observed chimpanzees at Ngogo in Kibale National Park, Uganda, over 12 months from 24 August 30, 2014 to August 30, 2015. Located in the center of the forest, the Ngogo study site is surrounded by other chimpanzee communities and covered by mature rainforest interspersed between areas of regenerating forest and grasslands (Struhsaker, 1997). The Ngogo chimpanzee community is large. For most of the study period, it consisted of 193 individuals, including 31 adult males, 23 adolescent males, 63 adult females, 15 adolescent females, 10 juvenile males, 5 juvenile females, and 46 infants. 
Subjects were ten middle and late adolescent males (12-16 years old) and eight young adult males (17-21 years old). These age categories correspond to physical and social milestones in male chimpanzee development and are based on previous studies conducted on chimpanzees at Gombe National Park and Mahale Mountains National Park in Tanzania and at Ngogo (Goodall, 1983, 1986; Kawanaka, 1989; Sandel et al., 2017). Although adolescence in male chimpanzees begins around 8-10 years of age, when their testes start to enlarge, we restricted study to individuals who had reached middle and late adolescence, as this is the time males begin to travel independently from their mothers on a regular basis (Pusey, 1983, 1990). Goodall (1983, p. 4) notes: "In behavioral terms, some males may not reach social maturity until 16 or even 17 years old." Because the two 16-year-old males in our sample were not involved in dominance interactions with their peers (Sandel et al., 2017), we classified them as adolescents.

\section{3 | Behavioral observations}

Sandel collected observations of male social behavior via continuous focal animal sampling (Altmann, 1974) during focal follows that lasted $1 \mathrm{hr}$. The time that subjects were out of sight was also recorded. Because chimpanzees live in fission-fusion societies, not all males were available for observation every day. We equalized the number of focal follows by rotating through subjects opportunistically, prioritizing males who had been observed less often than others cumulatively and during any given month. We also attempted to balance the focal follows across the time of day. Sandel followed chimpanzees from approximately 0730 to 1800 daily, and a majority of follows for each subject occurred in the late morning and early afternoon (starting between 1100 and 1200). Sandel conducted 812 focal follows representing $773.5 \mathrm{hr}$ of continuous observation (mean hours/individual $\pm S D$ : $43.1 \pm 3.1 \mathrm{hr}$, range: $38-50 \mathrm{hr}, \mathrm{N}=18$ males).

Sandel collected data on three behaviors (a) association in the same subgroup; (b) spatial proximity of $\leq 5 \mathrm{~m}$; and (c) grooming. Males who encountered the focal subject (i.e., were in visual range to the observer) for any period during hour-long focal follows were scored in association with the focal subject (cf. Mitani et al., 2002). Individuals in proximity $(\leq 5 \mathrm{~m})$ to the focal subject were recorded during instantaneous point samples made at 10-min intervals. The amount of grooming given and received by focal subjects was recorded continuously to the nearest second.

\subsection{Assessing social bonds}

Affiliative relationships between chimpanzees are manifest in three main behaviors: association, proximity, and grooming. Chimpanzees exhibit fission-fusion social dynamics, such that all individuals within the community, at least in East Africa, are never together at the same time, and instead split up into subgroups that change throughout the day. "Association" refers to individuals that are in a subgroup together. To some extent, patterns of association reflect who prefers to be with whom resulting from individual social decisions, such as traveling together (Newton-Fisher, 2002). However, patterns of association also reflect more passive processes that do not reflect social affinities, such as when individuals come together to feed at adjacent trees or the same large tree. As they associate with others, chimpanzees spend time in close spatial proximity to certain individuals, which provides another indication of partner preference. Being within five meters of another chimpanzee reflects a degree of tolerance that may not be required for association alone. Finally, grooming is frequently used to assess social bonds between primates (Dunbar, 2010; Silk, Cheney, \& Seyfarth, 2013), including male chimpanzees, who groom a small subset of individuals in their group (Mitani, 2009; Watts, 2000). Grooming is often considered the strongest indicator of bonds, as it requires even greater levels of effort and tolerance than does proximity, especially as pairs groom vulnerable areas, such as faces and genitalia.

We analyzed association, proximity, and grooming interactions separately. While some researchers combine different affiliative behaviors into a single index (Sapolsky, Alberts, \& Altmann, 1997; Silk, Altmann, \& Alberts, 2006), each behavior may reflect different aspects of relationships (Hirsch, Stanton, \& Maldonado, 2012; Schoof $\&$ Jack, 2014), and combining them may not accurately describe the bonds between animals (Lehmann, Korstjens, \& Dunbar, 2007). In this study, association, proximity, and grooming were correlated with one another, but there was variability (association vs. proximity: Pearson's correlation $=0.641,95 \%$ confidence interval: $0.602-0.677$; association vs. grooming: Pearson's correlation $=0.294$, $95 \%$ confidence interval: 0.234-0.351; proximity vs. grooming: Pearson's correlation $=0.555, \quad 95 \%$ confidence interval: 0.509-0.598; Figure S1). This variability suggests that each behavior may provide independent insights into the nature of social relationships.

\section{5 | Defining social bonds}

There is no consensus on how to define social bonds between individuals (Dunbar \& Shultz, 2010; Silk et al., 2013; Thompson, 2019; Whitehead, 2008). There are many aspects to any relationship between two individuals, including frequency of interactions, the different types of behavior engaged in by pairs, the symmetry of relationships, and how long they last (Silk et al., 2013). How often a pair interacts or the total duration of time spent together is a key measure, and pairs that spend more time than do the average pair are often considered bonded (Silk et al., 2013). We used this metric of social bonds.

We computed dyadic association by dividing the total number of focal follows during which pairs of males were together by the number of times the focal subject was followed. Dyadic proximity was calculated as the total number of instantaneous samples in which pairs of males were $\leq 5 \mathrm{~m}$ apart, divided by the total number of instantaneous samples for the focal subject. Dyadic grooming was 
calculated as the total minutes pairs of males spent grooming over the course of the year, divided by the observation hours for the focal subject.

To determine whether adolescent male chimpanzees exhibit bonds, we compared their relationships with other males to those of young adults. Adult male chimpanzees are known to form strong social bonds. Thus, we considered the strongest relationships formed by young adult males with other adult males (including young adults) to be good representations of social bonds. For each of the three measures of dyadic behavior, we considered the pairs in the top $10 \%$ of the distribution of young adult-adult dyads to be bonded for that behavior (Figure 1a), corresponding to the definition of bonded pairs used in prior studies (Schoof \& Jack, 2014; Silk et al., 2006). We then classified dyads that socialized at a rate that met oxceeded the absolute value of the top $10 \%$ threshold identified in young adultadult dyads as bonded for that particular dyadic behavior.
We determined for each behavior how many pairs of young adults had bonds with adolescent or adult males (ranging in age from 8 to over 50 years), and how many pairs of adolescents had bonds with adolescent or adult males. We then tallied the total number of bonds in each behavior for each dyad and compared the number of adultlike bonds formed by adolescents to those of young adults.

When constructing these distributions, we took the perspective of focal subjects by calculating dyadic association, proximity, and grooming rates from data collected while they were observed. We included relationships between the 18 focal subjects and 53 adolescent and adult males ranging in age from 8 to over 50 years ( $N=936$ total dyads). Adolescents could, therefore, appear as the partner of another focal subject in dyads. Because we took the perspective of the focal subject, when the focal subject in a dyad was an adolescent and he exhibited a bond with a young adult, we classified the bond as one by an adolescent. Conversely, when the (a)

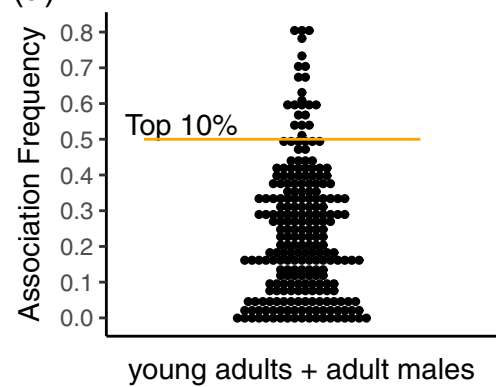

(d)

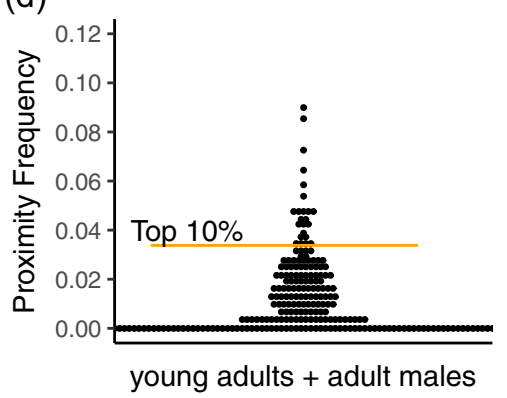

(g)

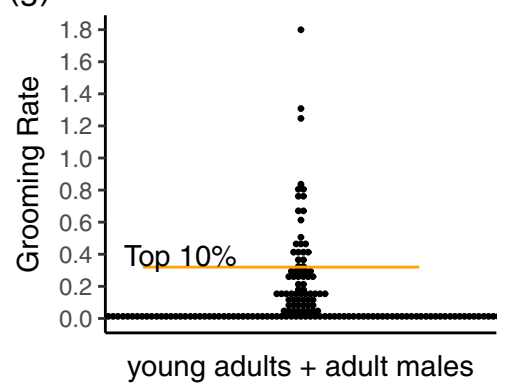

(b)

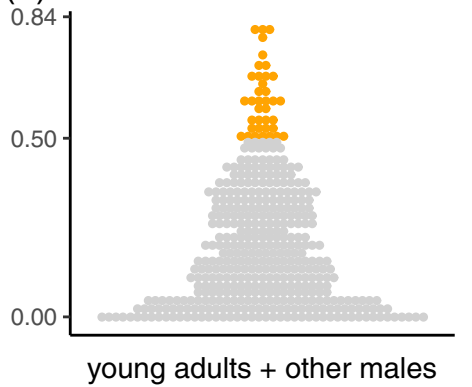

(e)

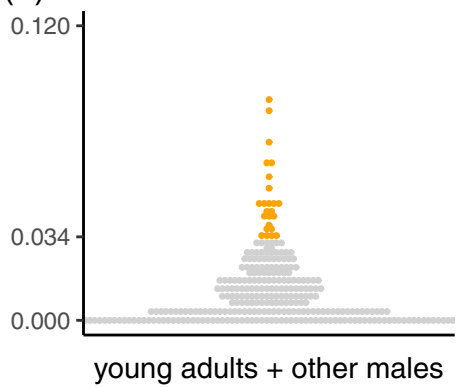

(h)

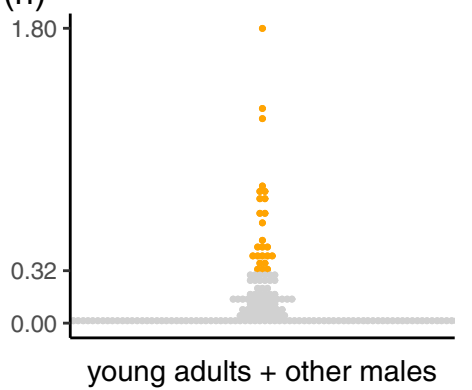

(c)

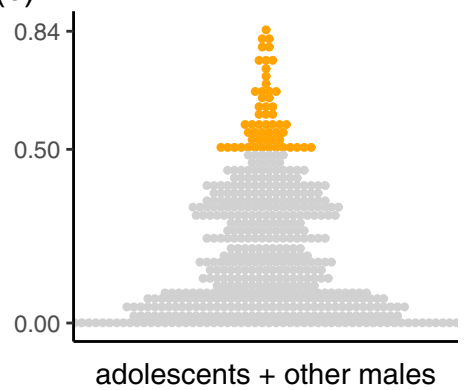

(f)

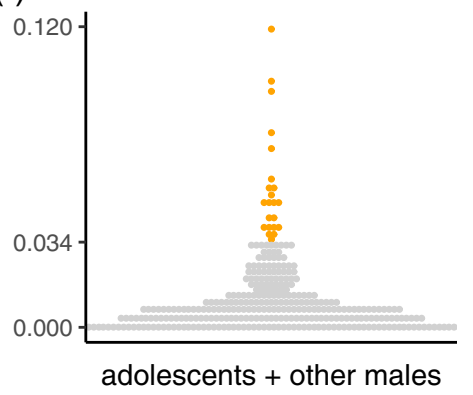

(i)

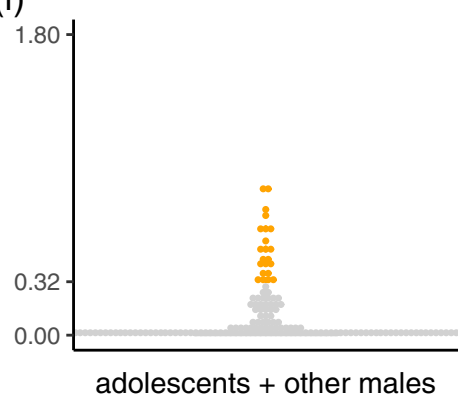

FIGURE 1 Social bonds of adolescent and young adult male chimpanzees, identified as the top 10\% of pairs in a sample of young adult males with other adult males separately for (a) association, (d) proximity, and (g) grooming. The value derived from the $10 \%$ threshold was used to classify bonds for young adults (b, e, h) and adolescents (c, f, i). Dyadic association frequencies represent the focal follows in association divided by total focal follows for the subject, proximity frequencies as the scans in proximity divided by total instantaneous point samples for the subject, and grooming rates as the total minutes grooming divided by hours of observation for the subject. Orange points are bonded pairs whereas gray points are those that fell in the lower $90 \%$ of the distribution based on the young adult-adult male distributions (a, $\mathrm{d}, \mathrm{g}$ ) 
focal subject was a young adult and he had a bond with an adolescent, we classified the bond as one by a young adult.

As a second means of assessing social bonds, we determined whether pairs of males displayed bonds based on all three of the measures of social behavior (Smuts, 1985; Whitehead, 2008). If a pair displayed bonds based on association, proximity, as well as grooming, we considered them to have a "triple bond."

\section{6 | Age}

Detailed observations of adult female chimpanzees, who are the mothers of our subjects, were initiated in 2001 at Ngogo (Langergraber, Mitani, \& Vigilant, 2009; Wakefield, 2008). Thus, our focal subjects, who were adolescents and young adults in 2014 , were identified as infants or juveniles, and their birth dates are known to within 1 month $(N=7)$ to 1 year $(N=11$; Sandel et al., 2017). Because the continuous study of chimpanzees at Ngogo began in 1995, the exact ages of many adults are unknown. Their ages have been estimated to the nearest year based on their physical appearance, behavior, and genetically established pedigrees (Wood, Watts, Mitani, \& Langergraber, 2017).

We created an age difference variable for each dyad by computing the absolute value of the difference between the age of the focal subject and the age of the other individual. We also assessed whether adolescent and young adult males exhibited triple bonds with males in different age classes. We considered males peers if they were within 5 years in age because this corresponds with the average interbirth interval for females (Mitani, 2009). Each focal subject in the sample had an average of 20 peers $(S D=2)$, with peers represented in 359 dyads. We defined potential male partners as old if they were $\geq 35$ years (Moeller et al., 2016). Based on this criterion, there were eight old males (mean age $\pm S D=43 \pm 5$ years, range: 38-53 years) in 144 dyads.

\section{7 | Kinship}

Kin relationships of our study subjects were based on analyses of 19-44 autosomal microsatellites in addition to mitochondrial DNA as described in previous work (Langergraber et al., 2007, 2009; Langergraber, Mitani, Watts, \& Vigilant, 2013). Father-son ( $N=11)$ and mother-son $(N=16)$ dyads were determined with likelihoodbased parentage analyses (Langergraber et al., 2013; Marshall, Slate, Kruuk, \& Pemberton, 1998). Pairs of males were assigned as maternal brothers, paternal brothers, fathers and sons, or "unrelated" (i.e., all other types of dyads, including distant relatives such as uncle-nephew and first cousins). Dyads that had the same father as identified through paternity analysis were classified as paternal brothers; dyads that had the same mother as identified through behavioral observations and maternity analysis were classified as maternal brothers. The father was identified for all adolescent and young adult males, and the mother was identified for 16 of the 18 focal subjects. For the two males without known mothers, we compared their mitochondrial DNA haplotype to other males without known mothers $(N=7)$, and we could exclude the possibility of their being maternal brothers based on their haplotype for all but three pairs of males. For these three dyads, their status as maternal brothers was unknown. One pair shared a similar part of their range and the younger male had followed the older since he was a young adolescent. We classified this pair as maternal brothers and assigned the other two dyads with unknown maternal brother status as unrelated. To test the effect of this assumption, we conducted additional analyses with all three assigned as unrelated or all three assigned as maternal brothers (see Section 2.8).

Twelve adolescent and young adult males had maternal brothers who were adolescents or adults, involving 15 dyads. All maternal brothers were half-siblings except for one pair who were full siblings. Fourteen adolescent and young adult males had paternal brothers who were adolescents or adults, involving 34 dyads. This total excluded the full sibling pair, who we classified as maternal brothers for purposes of the following analyses. Eleven adolescent and young adult males had living fathers, which included six different males.

\section{8 | Statistical analyses}

To determine whether adolescent males exhibited social bonds with other males as often as did young adults, we compared the mean number of bonds displayed by males of both age classes using a Welch unequal variance $t$-test. We used the number of times adolescents exhibited triple bonds with others as a second means to evaluate how frequently they developed bonds compared to young adults. Here we calculated the number of times adolescent males exhibited bonds based on all three behaviors divided by the total number of triple bonds formed by adolescent and young adult subjects. We used the resulting percentage as a test statistic. High values indicated that adolescents frequently had strong bonds, while low values reflected the opposite. Because pairs were the unit of analysis, individuals appeared multiple times, and data points were not independent. In addition, there were ten adolescents and eight young adults in the sample, so the former had a higher probability of being in bonded pairs. To generate a null distribution of the percentage of strong bonds formed by adolescents, we drew pairs of males at random without replacement from the pool of 936 total dyads, holding the observed number of triple bonds constant. We then calculated the number of times adolescents appeared in the samples of triple bonds relative to the total number of triply-bonded pairs and used this as one datum in the null distribution. We repeated this process 10,000 times to generate a $95 \%$ confidence interval. We compared the observed test statistic to the null distribution to evaluate whether adolescents exhibited strong bonds as frequently as did young adults.

To assess the effects of age, age difference, and kinship on the social bonds of adolescent and young adult males, we ran three generalized linear mixed models, with the association, proximity, and 
grooming between pairs of males as the outcome variables. Association and proximity were kept as counts. Grooming was measured as a continuous variable, the duration of time spent grooming. For the association model, we added the log number of hour-long focal follows on the subject as a fixed effect to control for variation in observation time. In the proximity and grooming models, we excluded dyads that never associated and added the log number of times each pair was in the association as a fixed effect to control for variation in opportunities to interact. Thus, proximity and grooming were assessed relative to association time. Results should be interpreted with this in mind. Fixed effects were the age of the subject, the dyads' kinship (i.e., maternal brothers, paternal brothers, father-son, or unrelated), the age of the other male, and the absolute value of the age difference between the pair. The identity of subjects and the other individual in the dyad were included as random effects. We set a negative binomial error distribution with the "glmmADMB" package (Fournier et al., 2012; Skaug, Fournier, Bolker, Magnusson, \& Nielsen, 2016) in R (R Core Team 2015). To test the influence of our assumptions about the kinship of the three pairs of males whose status as maternal brothers was unknown, we conducted two additional models (a) with the unknown pairs classified as unrelated, and (b) with the three pairs as maternal brothers. Doing so did not qualitatively change the results.

In a final series of analyses, we calculated the percentage of triple bonds between adolescents and young adults and (a) peers and old males and (b) maternal brothers, paternal brothers, fathers, and unrelated males. We conducted the same randomization procedure described above to generate null distributions and used these to assess the effects of relatedness and age on the formation of bonds.

\section{3 | RESULTS}

\subsection{When do social bonds form?}

Adolescent male chimpanzees exhibited social bonds with other males (Figure 1), and they did so as frequently as did young adult males (Figure 2). The number of bonds based on associations did not differ between adolescents (mean $\pm S D=4.4 \pm 3.5$ ) and young adults (mean $=4.1 \pm 3.6$, Welch two-sample $t$-test: $t_{14.957}=0.165 ; p=.872$ ) nor did the number of proximity bonds (adolescent mean $\pm S D=2.2$ \pm 2.3 , young adult mean $\pm S D=2.6 \pm 1.7, t_{15.917}=-0.452, p=.657$ ) or grooming bonds (adolescent mean $\pm S D=2.2 \pm 2.2$, young adult mean $\pm S D=3.0 \pm 3.0, t_{12.452}=-0.627 ; p=.542$ ). We found similar results when we examined age as a continuous variable. Age did not predict the number of bonds exhibited by individuals for association (linear regression: $F(1,16)=0.06$, age coefficient $=-0.08 ; p=.80$ ) proximity $(F(1,16)<0.001$, age coefficient $=-0.003 ; p=.99)$, or grooming $(F(1,16)=0.28$, age coefficient $=0.131 ; p=.61)$.

All adolescent males had at least one type of social bond with another male. Nine of ten adolescents had an association bond. Seven of ten adolescents exhibited proximity bonds and seven displayed grooming bonds. Only two adolescent males did not have proximity or grooming bonds (Figure 2). Adolescent males displayed 44 associations bonds, 22 proximity bonds, and 22 grooming bonds with other males. These values underestimate the true number of social bonds involving adolescents because bonds between them and young adults, calculated from when the latter were targets of observation, were not included in these totals. Of the 33 association bonds exhibited by young adults, 11 were with adolescent males. Most proximity $(95 \%=20 / 21)$ and grooming bonds $(92 \%=22 / 24)$ formed by young adults, however, were with adults rather than with adolescents.

Some pairs of males exhibited particularly strong bonds based on the three different measures of behavior, and adolescents did so as frequently as did young adults. Adolescents were involved in $70 \%$ (7/10) of triple bonds (expected 95\% confidence interval (Cl): 20-80\%). Because adolescents and young adult males did not differ in the number or strength of bonds that they had with others, we combined the two age groups in subsequent analyses to increase the power of statistical tests.
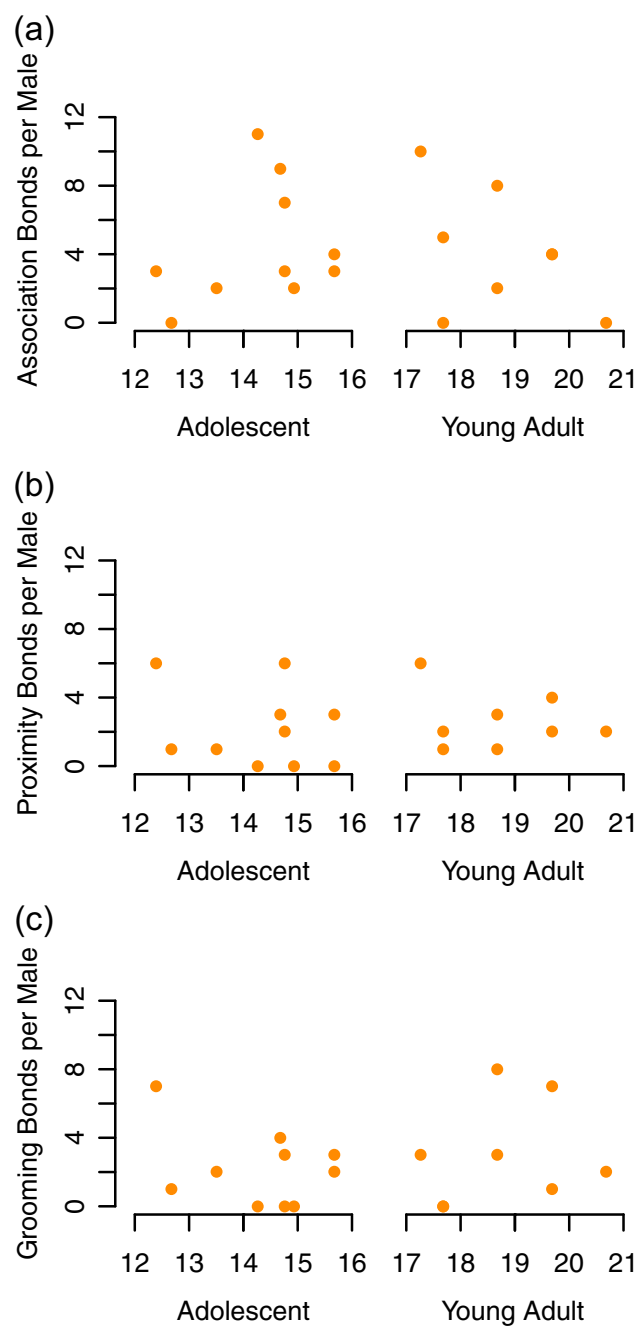

FIGURE 2 Number of social bonds ( $a, b$, and c) of adolescent and young adult male chimpanzees. X-axis represents age in years at the mid-point of the study 


\subsection{Effect of age and age difference}

To determine the effects of age and kinship, we ran multiple regression models with the age of the focal subject, the age of the partner, the age difference between the focal subject and the partner, the dyad's kin category, and how often the dyad associated together as predictors. Adolescent and young adult males associated more often with older rather than younger males (Table 1). Adolescent and young adult males also spent more time in proximity and groomed more with older than younger males when in association (Table 1). Some of the strongest bonds involved the eight oldest adult males, who were 38 years and older. These males were involved in $40 \%$ of all of the triple bonds, a percentage that approached the outer bound of what would be expected by chance (expected $\mathrm{Cl}$ : $0-40 \%)$. These old males were also involved in $12 \%(9 / 77)$ of association bonds (including three different old males), 35\% (15/43) of proximity bonds (including six different old males), and 35\% (16/46) of grooming bonds (including all eight old males; Table S1).

Although adolescents and young adults bonded with old males, relationships also occurred with peers. Controlling for the other male's age and time spent in the association, adolescents and young adults spent more time in proximity to and grooming with males similar in age than males who were older or younger (Table 1). Peers were involved in $42 \%(32 / 77)$ of association bonds, $26 \%(11 / 43)$ of proximity bonds, and $24 \%$ (11/46) of grooming bonds (Table S1). Some bonds were particularly strong, with $30 \%$ of triple bonds between peers, but this did not exceed chance expectations based on the number of peers available.

\subsection{Effect of kinship}

Controlling for the age of the other male and age difference between the dyad, adolescent and young adult males were more likely to socialize with individuals in some kin categories compared with unrelated males (Figure 3). Adolescent and young adult males associated with maternal brothers more often than they did with unrelated males. They also spent more time in proximity with maternal brothers than they did with unrelated males given how often they associated together (Table 1). Maternal brothers formed

TAB LE 1 Models of association, proximity, and grooming between adolescent/young adult males and other males predicted by kinship, age of the subject, age of the partner, and age difference of the pair.

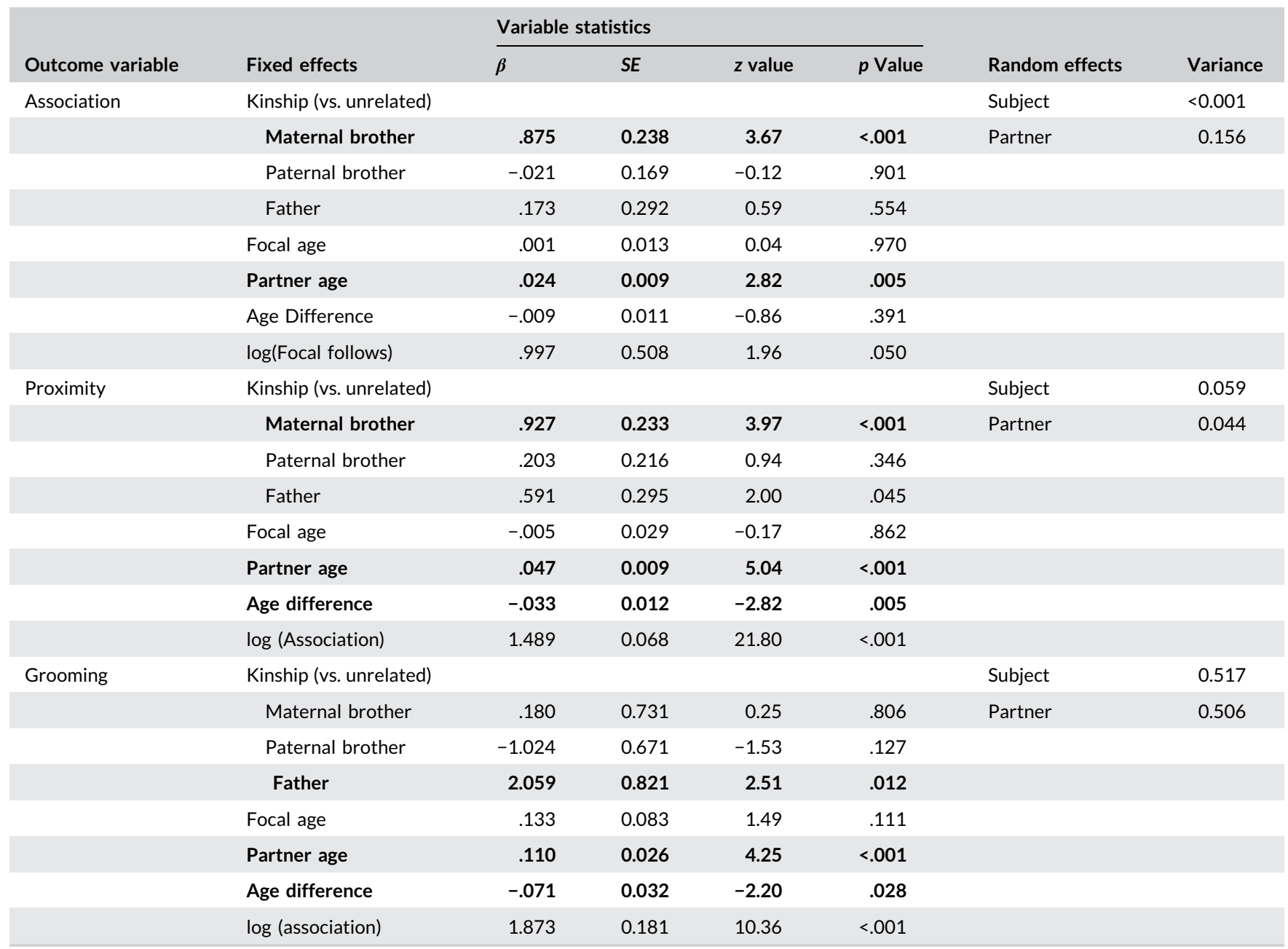

The variables in bold typeface represent significant predictors $(p<.05)$. 
(a)

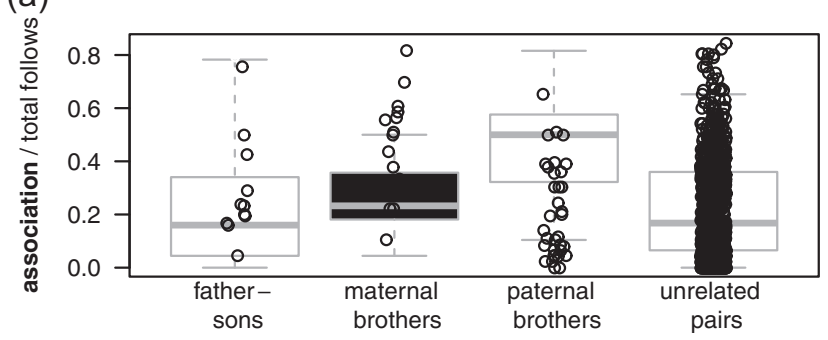

(b)

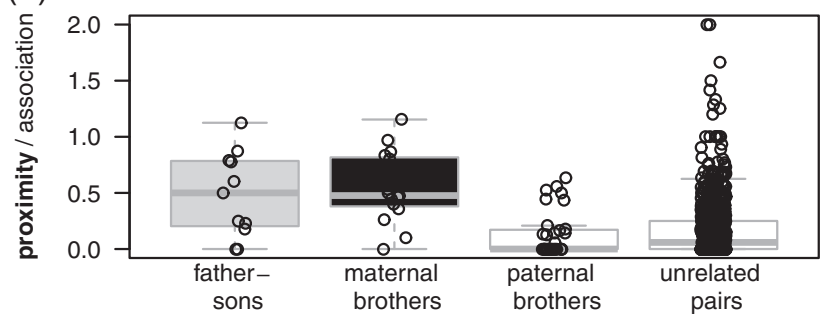

(c)

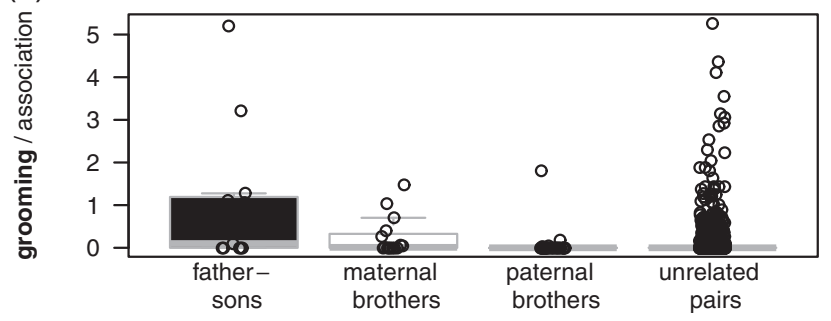

FIGURE 3 Boxplots of affiliation measures by kinship class. (a) Association time relative to total observation time. (b) Proximity time relative to total association time. (c) Grooming time relative to total association time. Black boxes indicate strong effects of that kin category compared to unrelated dyads, and light gray boxes indicate weaker effects as indicated by statistical tests conducted using multiple regression of relatedness, age, and age difference in Table 1

some of the strongest bonds with each other, displaying a significantly higher than expected number of triple bonds (30\% of bonds; expected Cl: 0-10\%). Although maternal kinship had a strong effect on social behavior, its effect was not uniform. Adolescents and young adults did not groom more frequently with their maternal brothers than with unrelated males given how often they associated together (Table 1). In addition, seven of the 15 males did not form any type of social bond with their maternal brothers. In four of these seven cases, adolescent and young adult males did not have bonds with their younger adolescent brothers. In two cases they did not have bonds with their high-ranking, prime-aged adult brothers. Finally, one adolescent did not have a bond with his low-ranking, prime-aged adult brother.

In contrast to maternal kinship, there was no bias to groom with, spend time in proximity to, or associate with paternal brothers (Table 1). There were no triply bonded paternal brothers (expected Cl: 0-20\%). Two males had association bonds with their paternal brothers, no paternal brothers had proximity bonds, and one male had a grooming bond with his paternal brother.

Although adolescents and young adult male chimpanzees did not preferentially socialize with their paternal brothers, they did do so with their fathers. Controlling for age, age difference, and association time, adolescent and young adult male chimpanzees groomed more with their fathers than with unrelated males (Table 1). There was also a trend for young males to spend more time in proximity to fathers than unrelated males (Table 1). Since all models included kinship and age as fixed effects, the preference to socialize with fathers was present despite controlling for the age of the partner and vice versa. While males transitioning to adulthood spent considerable time grooming with their fathers, they did not preferentially associate with their fathers compared to unrelated males (Figure 3). Some father-son pairs displayed particularly strong bonds. Of the 11 males who had living fathers, three had grooming bonds with them. Two males had their fathers as their top grooming partners. No male had a triple bond with his father (expected $\mathrm{Cl}: 0-10 \%$ ), but one exhibited proximity and grooming bonds with his father. Four of the 11 males formed at least one type of bond with their father, based on association, proximity, or grooming (Table S1), including one male who had an association bond and another who had a proximity bond with his father.

\section{4 | DISCUSSION}

Adolescent male chimpanzees exhibited social bonds with other males. They did not differ from young adults in the number or strength of bonds. Consistent with previous research on male chimpanzees (Langergraber et al., 2007; Mitani, 2009; Mitani et al., 2002), adolescent and young adult males bonded with their maternal brothers and peers but not with their paternal brothers. Many of the strongest relationships were with unrelated males, including old males. Unexpectedly, they frequently groomed with their fathers.

The function of social bonds among adolescents is unclear. As adults, male chimpanzees establish long-term social bonds with each other, in part, to help acquire and maintain high dominance rank (Mitani, 2009; Nishida, 2012). But adolescent male chimpanzees, unlike adults, do not form decided dominance relationships with one another (Sandel et al., 2017). Adolescents, therefore, must exhibit bonds for reasons unrelated to immediate status competition. One possibility is that males use social bonds to buffer against the stress that they endure as adolescents. During infancy and juvenility, mothers are in near-constant contact with their sons, serving as their primary grooming partner and source of support (Pusey, 1983, 1990). The social lives of male chimpanzees change drastically during adolescence when they start to travel independently of their mothers, receive increased aggression from adult males, and remain at the periphery of subgroups, sometimes even after reaching adulthood (Kawanaka, 1989; Pusey, 1990). Prior studies indicate that social bonds mitigate stress, as assayed by glucocorticoid levels, in female baboons (Papio ursinus) and adult chimpanzees (Crockford et al., 2008; Wittig et al., 2008; Wittig et al., 2016). Adolescent male chimpanzees may use social bonds in the same way.

A second possibility is that adolescent male chimpanzees forge social bonds to facilitate their entry into the adult social network. 
Our results show that adolescent and young adult males develop association and proximity bonds with their older maternal brothers, who are likely allies, as they have a long history of contact and familiarity resulting from a shared relationship with their mothers (Pusey, 1983, 1990). Adolescent and young adult males did not frequently groom with their maternal brothers given how much they associated. Although male chimpanzees may be tolerant of their younger brothers and spend considerable time together, they do not necessarily form intimate grooming bonds. Instead, adolescent and young adult male chimpanzees may prioritize grooming with older males who are in a position to provide coalitionary support (Watts, 2002).

In addition to the bonds with maternal brothers, adolescent and young adult male chimpanzees exhibited some of their strongest social bonds with some unrelated males, who were relatively old. In general, old males are no longer involved in the competitive world of prime adults, although they remain socially integrated (Hosaka \& Huffman, 2015; Nishida, 2012). Adolescent and young adult males may be attracted to these "retired" males, who are more tolerant of young hangers-on than are prime adult males. The social behavior of other primates appears to change with age (Almeling, Hammerschmidt, Sennhenn-Reulen, Freund, \& Fischer, 2016), and across taxa, old males have sometimes been described as more relaxed than their younger selves (Dagg, 2009). Few studies have investigated old age in chimpanzees (Hosaka \& Huffman, 2015), but one study of captive chimpanzees found that old males were less aggressive than were young adults (Baker, 2000). If old males are no longer entrenched in the competitive world of adult male chimpanzees, they may make ideal partners for young males as they transition to adulthood.

In their relationships with old males, adolescent and young adult males appear to prefer certain males as social partners, including their fathers. Relationships with fathers were unexpected as prior studies considered it unlikely that chimpanzees are able to discriminate paternal relatives given their polygynandrous mating system (Langergraber et al., 2007; Wroblewski, 2010). Nevertheless, two previous studies found that fathers interact nonrandomly with their offspring. At the Taï National Park, Côte d'Ivoire, chimpanzee fathers did not preferentially associate, groom, or play with their offspring, but when adults did play with infants or juveniles, and both offspring and unrelated youngsters were present, fathers played longer with their offspring than they did with unrelated individuals (Lehmann et al., 2006). Similarly, at Gombe National Park, Tanzania, fathers preferentially associated with the mothers of their offspring (Murray et al., 2016), a pattern that also occurs at Ngogo (Langergraber et al., 2013). In addition, infants at Gombe, although spending very little time interacting with adult males, groomed and played more often with their fathers than nonrelatives (Murray et al., 2016). Our observations complement these prior findings. Results of previous studies have been derived from observations of infants and juveniles, whose affiliative behavior is mediated, if not controlled entirely, by their mothers (Murray et al., 2014; Pusey, 1983). In contrast, the relationships we documented involved older individuals, adolescents, and young adults, who are acting independently of their mothers.
How do the father-son grooming bonds that we have described here develop? One possibility emerges from the social, spatial, and reproductive behavior of the Ngogo chimpanzees. At Ngogo, female and male chimpanzees form social and spatial subgroups (Langergraber et al., 2009; Mitani \& Amsler, 2003; Wakefield, 2008). Males gain a reproductive advantage with subgroup females (Langergraber et al., 2013), which creates opportunities for sons to interact frequently with their fathers as they grow up. Thus, the bonds forged between fathers and sons later in life may arise early during development via familiarity, as has been suggested in other primate species (Berenstain, Rodman, \& Glenn-Smith, 1981; Huchard et al., 2010; Huchard et al., 2013; Kerhoas et al., 2016; Langos et al., 2013; Moscovice, Heesen, Di Fiore, Seyfarth, \& Cheney, 2009; Palombit, Seyfarth, \& Cheney, 1997; Rosenbaum, Hirwa, Silk, \& Stoinski, 2016). Testing this hypothesis will require further study. A second possibility is that adolescent and young adult males are attracted to high-ranking males (Rosenbaum, Hirwa, Silk, Vigilant, \& Stoinski, 2015) or formerly high-ranking males. High status is positively related to reproduction in chimpanzees; high-ranking males, therefore, have a stronger chance of fathering infants than do low-ranking males (e.g., Boesch et al., 2006; Wroblewski et al., 2009). Additional longitudinal study will be required to investigate the roles of familiarity and male dominance rank on the formation of father-son bonds in chimpanzees.

Future studies should also investigate whether the relationships with fathers and social bonds in general emerge earlier in development. We found that by age 12 years, during middle adolescence, male chimpanzees exhibit social bonds with males that resemble those of young adult male chimpanzees. However, it is possible that such relationships develop even earlier. Whether early adolescents and juveniles develop adult-like social bonds or preferences for certain individuals remains to be explored.

Taken together, our findings reveal that adolescent male chimpanzees maintain social bonds with other males and suggest that the bonds forged between males and their maternal brothers, old males, and fathers may play an important role during the transition to adulthood. If bonds with fathers are characteristic of chimpanzees generally, studying the mechanism and function of these relationships may offer insight into how paternal care in humans, associated with pair-bonding and relatively exclusive mating, could have evolved from phylogenetic building blocks already present in a chimpanzee-like social and mating system (Chapais, 2008).

\section{ACKNOWLEDGEMENTS}

The research was approved by the Uganda Wildlife Authority and the Uganda National Council for Science and Technology and supported by the Makerere University Biological Field Station. Broader impacts of this project included conservation education and capacity building at Kyakagunga Primary School, Kamwenge District, Uganda. A. A. Sandel was supported by The Leakey Foundation, the University of Michigan, the Nacey-Maggioncalda Foundation, and the National 
Science Foundation (1540259 and F031543). J. C. Mitani and K. E. Langergraber are currently supported by NIH RO1AG049395. Nathan Chesterman provided assistance and friendship in the field. For additional support in the field, the authors thank David Watts, Sam Angedakin, Alfred Tumusiime, Ambrose Twineomujuni, Godfrey Mbabazi, Lawrence Ndangizi, Rachna Reddy, and the late Jerry Lwanga. For statistical advice, the authors thank the Center for Statistical Consulting and Research at the University of Michigan, especially Kerby Shedden and Josh Errickson from 2015 to 2017. For comments on an earlier version of this manuscript, the authors thank Marina Cords, two anonymous reviewers, and Karen Bales. For helpful advice and feedback during the development of this project, the authors thank Jacinta Beehner, Thore Bergman, Anne Pusey, Brent Pav, Rachna Reddy, Nathan Chesterman, Andy Marshall, Sam Patterson, Bethany Hansen, Ben Finkel, Barb Smuts, Michael Sandel, Kiku Adatto, and additional friends, family, and colleagues of A. A. Sandel.

\section{DATA AVAILABILITY STATEMENT}

The data that support the findings of this study are available on request from the corresponding author and are summarized as a supplementary file to this manuscript.

\section{ORCID}

Aaron A. Sandel (D) http://orcid.org/0000-0003-3060-5310 John C. Mitani (D) http://orcid.org/0000-0001-7042-5854

\section{REFERENCES}

Alberts, S. C. (2019). Social influences on survival and reproduction: Insights from a long-term study of wild baboons. Journal of Animal Ecology, 88(1), 47-66. https://doi.org/10.1111/1365-2656.12887

Almeling, L., Hammerschmidt, K., Sennhenn-Reulen, H., Freund, A. M., \& Fischer, J. (2016). Motivational shifts in aging monkeys and the origins of social selectivity. Current Biology, 26(13), 1744-1749. https://doi. org/10.1016/j.cub.2016.04.066

Altmann, J. (1974). Observational study of behavior: Sampling methods. Behaviour, 49(3-4), 227-266. https://doi.org/10.1163/156853974X00534

Amici, F., Kulik, L., Langos, D., \& Widdig, A. (2019). Growing into adulthood -a review on sex differences in the development of sociality across macaques. Behavioral Ecology and Sociobiology, 73(2), 18. https://doi. org/10.1007/s00265-018-2623-2

Baker, K. C. (2000). Advanced age influences chimpanzee behavior in small social groups. Zoo Biology, 19(2), 111-119. https://doi.org/10. 1002/1098-2361(2000)19:2<111::AID-ZOO2>3.0.CO;2-5

Berenstain, L., Rodman, P. S., \& Glenn-Smith, D. (1981). Social relations between fathers and offspring in a captive group of rhesus monkeys (Macaca mulatta). Animal Behaviour, 29(4), 1057-1063. https://doi.org/ 10.1016/S0003-3472(81)80058-9

Boesch, C., Kohou, G., Néné, H., \& Vigilant, L. (2006). Male competition and paternity in wild chimpanzees of the Taï forest. American Journal of Physical Anthropology, 130(1), 103-115. https://doi.org/10.1002/ ajpa.20341
Chapais, B. (2008). Primeval kinship: How pair-bonding gave birth to human society. Cambridge, MA: Harvard University Press.

Charpentier, M. J. E., Peignot, P., Hossaert-McKey, M., \& Wickings, E. J. (2007). Kin discrimination in juvenile mandrills, Mandrillus sphinx. Animal Behaviour, 73(1), 37-45. https://doi.org/10.1016/j.anbehav. 2006.02.026

Charpentier, M. J. E., Van Horn, R. C., Altmann, J., \& Alberts, S. C. (2008). Paternal effects on offspring fitness in a multimale primate society. Proceedings of the National Academy of Sciences, 105(6), 1988-1992. https://doi.org/10.1073/pnas.0711219105

Crockford, C., Wittig, R., Whitten, P., Seyfarth, R., \& Cheney, D. (2008). Social stressors and coping mechanisms in wild female baboons (Papio hamadryas ursinus). Hormones and Behavior, 53(1), 254-265. https:// doi.org/10.1016/j.yhbeh.2007.10.007

Dagg, A. I. (2009). The social behavior of older animals. Baltimore, MD: The Johns Hopkins University Press.

Dunbar, R., \& Shultz, S. (2010). Bondedness and sociality. Behaviour, 147(7), 775-803. https://doi.org/10.1163/000579510X501151

Dunbar, R. I. M. (2010). The social role of touch in humans and primates: Behavioural function and neurobiological mechanisms. Neuroscience \& Biobehavioral Reviews, 34(2), 260-268. https://doi.org/10.1016/j. neubiorev.2008.07.001

Fournier, D., Skaug, H., Ancheta, J., lanelli, J., Magnusson, A., Maunder, M., ... Sibert, J. (2012). AD Model Builder: Using automatic differentiation for statistical inference of highly parameterized complex nonlinear models. Optimitzation Methods and Software, 27(2), 233-249. https:// doi.org/10.1080/10556788.2011.597854

Godoy, I., Vigilant, L., \& Perry, S. E. (2016). Cues to kinship and close relatedness during infancy in white-faced capuchin monkeys, Cebus capucinus. Animal Behaviour, 116, 139-151. https://doi.org/10.1016/j. anbehav.2016.03.031

Goodall, J. (1983). Population dynamics during a 15 year period in one community of free-living chimpanzees in the Gombe National Park, Tanzania. Zeitschrift für Tierpsychologie, 61(1), 1-60. https://doi.org/10. 1111/j.1439-0310.1983.tb01324.x

Goodall, J. (1986). The chimpanzees of Gombe: patterns of behavior. Cambridge, MA: Harvard University Press.

Hirsch, B. T., Stanton, M. A., \& Maldonado, J. E. (2012). Kinship shapes affiliative social networks but not aggression in ring-tailed coatis. PLOS One, 7(5), 1-9. https://doi.org/10.1371/journal.pone.0037301

Hosaka, K., \& Huffman, M. (2015). Gerontology. In M. Nakamura, K. Hosaka, N. Itoh \& K. Zamma (Eds.), Mahale chimpanzees: 50 Years of research (pp. 326-339). Cambridge, UK: Cambridge University Press.

Huchard, E., Alvergne, A., Féjan, D., Knapp, L. A., Cowlishaw, G., \& Raymond, M. (2010). More than friends? Behavioural and genetic aspects of heterosexual associations in wild chacma baboons. Behavioral Ecology and Sociobiology, 64(5), 769-781. https://doi.org/ 10.1007/s00265-009-0894-3

Huchard, E., Charpentier, M. J., Marshall, H., King, A. J., Knapp, L. A., \& Cowlishaw, G. (2013). Paternal effects on access to resources in a promiscuous primate society. Behavioral Ecology, 24(1), 229-236. https://doi.org/10.1093/beheco/ars158

Kawanaka, K. (1989). Age differences in social interactions of young males in a chimpanzee unit-group at the Mahale Mountains National Park, Tanzania. Primates, 30(3), 285-305. https://doi.org/10.1007/ bf02381256

Kawanaka, K. (1993). Age differences in spatial positioning of males in a chimpanzee unit-group at the Mahale Mountains National Park, Tanzania. Primates, 34(3), 255-270. https://doi.org/10.1007/ bf02382620

Kerhoas, D., Kulik, L., Perwitasari-Farajallah, D., Agil, M., Engelhardt, A., \& Widdig, A. (2016). Mother-male bond, but not paternity, influences male-infant affiliation in wild crested macaques. Behavioral Ecology and Sociobiology, 70(8), 1117-1130. https://doi.org/10.1007/s00265-0162116-0 
Langergraber, K., Mitani, J., \& Vigilant, L. (2007). The limited impact of kinship on cooperation in wild chimpanzees. Proceedings of the National Academy of Sciences, 104(19), 7786-7790.

Langergraber, K., Mitani, J., Watts, D., \& Vigilant, L. (2013). Male-female socio-spatial relationships and reproduction in wild chimpanzees. Behavioral Ecology and Sociobiology, 67(6), 861-873. https://doi.org/10. 1007/s00265-013-1509-6

Langergraber, K. E., Mitani, J. C., \& Vigilant, L. (2009). Kinship and social bonds in female chimpanzees (Pan troglodytes). American Journal of Primatology, 71(10), 840-851. https://doi.org/10.1002/ajp.20711

Langos, D., Kulik, L., Mundry, R., \& Widdig, A. (2013). The impact of paternity on male-infant association in a primate with low paternity certainty. Molecular Ecology, 22(13), 3638-3651. https://doi.org/10. 1111/mec.12328

Lehmann, J., Fickenscher, G., \& Boesch, C. (2006). Kin biased investment in wild chimpanzees. Behaviour, 143(8), 931-955. https://doi.org/10. 1163/156853906778623635

Lehmann, J., Korstjens, A. H., \& Dunbar, R. I. M. (2007). Group size, grooming and social cohesion in primates. Animal Behaviour, 74(6), 1617-1629. https://doi.org/10.1016/j.anbehav.2006.10.025

Marshall, T., Slate, J., Kruuk, L., \& Pemberton, J. (1998). Statistical confidence for likelihood-based paternity inference in natural populations. Molecular Ecology, 7(5), 639-655. https://doi.org/10. 1046/j.1365-294x.1998.00374.x

Massen, J., Sterck, E., \& de Vos, H. (2010). Close social associations in animals and humans: Functions and mechanisms of friendship. Behaviour, 147(11), 1379-1412. https://doi.org/10.1163/000579510X528224

Mitani, J. (2009). Male chimpanzees form enduring and equitable social bonds. Animal Behaviour, 77(3), 633-640. https://doi.org/10.1016/j. anbehav.2008.11.021

Mitani, J., Watts, D., Pepper, J., \& Merriwether, D. (2002). Demographic and social constraints on male chimpanzee behaviour. Animal Behaviour, 64(5), 727-737. https://doi.org/10.1006/anbe.2002.4014

Mitani, J. C., \& Watts, D. P. (2001). Why do chimpanzees hunt and share meat? Animal Behaviour, 61(5), 915-924. https://doi.org/10.1006/ anbe.2000.1681

Mitani, J. C., \& Amsler, S. J. (2003). Social and spatial aspects of male subgrouping in a community of wild chimpanzees. Behaviour, 140(7), 869-884. https://doi.org/10.1163/156853903770238355

Moeller, A. H., Foerster, S., Wilson, M. L., Pusey, A. E., Hahn, B. H., \& Ochman, H. (2016). Social behavior shapes the chimpanzee panmicrobiome. Science Advances, 2(1), 1-6. https://doi.org/10.1126/ sciadv. 1500997

Moscovice, L. R., Heesen, M., Di Fiore, A., Seyfarth, R. M., \& Cheney, D. L. (2009). Paternity alone does not predict long-term investment in juveniles by male baboons. Behavioral Ecology and Sociobiology, 63(10), 1471-1482. https://doi.org/10.1007/s00265-009-0781-y

Murray, C. M., Stanton, M. A., Lonsdorf, E. V., Wroblewski, E. E., \& Pusey, A. E. (2016). Chimpanzee fathers bias their behaviour towards their offspring. Royal Society Open Science, 3, 160441. https://doi.org/10. 1098/rsos.160441.

Murray, C. M., Lonsdorf, E. V., Stanton, M. A., Wellens, K. R., Miller, J. A., Goodall, J., \& Pusey, A. E. (2014). Early social exposure in wild chimpanzees: mothers with sons are more gregarious than mothers with daughters. Proceedings of the National Academy of Sciences, 111(51), 18189-18194.

Newton-Fisher, N. E. (2002). Ranging patterns of male chimpanzees in the Budongo forest, Uganda: Range structure and individual differences. In C. S. Harcourt \& B. Sherwood (Eds.), New perspectives in primate evolution and behaviour (pp. 287-308). Otley, West Yorkshire: Westbury Academic \& Scientific Publishing.

Nishida, T. (1979). The social structure of chimpanzees of the Mahale Mountains. In D. Hamburg \& E. McCown (Eds.), The great apes (pp. 73-122). Menlo Park, CA: The Benjamin/Cummings Publishing Company.
Nishida, T. (1983). Alpha status and agonistic alliance in wild chimpanzees (Pan troglodytes schweinfurthii). Primates, 24(3), 318-336. https://doi. org/10.1007/bf02381978

Nishida, T. (2012). Chimpanzees of the lakeshore: natural history and culture at Mahale. Cambridge, UK: Cambridge University Press.

Nishida, T., \& Hosaka, K. (1996). Coalition strategies among adult male chimpanzees of the Mahale Mountains, Tanzania. In W. McGrew, L. Marchant \& T. Nishida (Eds.), Great ape societies (pp. 114-134). Cambridge, UK: Cambridge University Press.

Palombit, R. A., Seyfarth, R. M., \& Cheney, D. L. (1997). The adaptive value of 'friendships' to female baboons: Experimental and observational evidence. Animal Behaviour, 54(3), 599-614. https://doi.org/10.1006/ anbe.1996.0457

Pfefferle, D., Kazem, A. J., Brockhausen, R. R., Ruiz-Lambides, A. V., \& Widdig, A. (2014). Monkeys spontaneously discriminate their unfamiliar paternal kin under natural conditions using facial cues. Current Biology, 24(15), 1806-1810. https://doi.org/10.1016/j.cub.2014.06.058

Pusey, A. (1983). Mother-offspring relationships in chimpanzees after weaning. Animal Behaviour, 31(2), 363-377. https://doi.org/10.1016/ s0003-3472(83)80055-4

Pusey, A. (1990). Behavioural changes at adolescence in chimpanzees. Behaviour, 115(3/4), 203-246.

R Core Team (2015). R: A language and environment for statistical computing. Vienna, Austria: R Foundation for Statistical Computing. https://www.R-project.org/

Riss, D., \& Goodall, J. (1977). The recent rise to the alpha-rank in a population of free-living chimpanzees. Folia Primatologica, 27, 134-151. https://doi.org/10.1159/000155784

Rosenbaum, S., Hirwa, J. P., Silk, J. B., \& Stoinski, T. S. (2016). Relationships between adult male and maturing mountain gorillas (Gorilla beringei beringei) persist across developmental stages and social upheaval. Ethology, 122(2), 134-150. https://doi.org/10.1111/ eth.12457

Rosenbaum, S., Hirwa, J. P., Silk, J. B., Vigilant, L., \& Stoinski, T. S. (2015). Male rank, not paternity, predicts male-immature relationships in mountain gorillas, Gorilla beringei beringei. Animal Behaviour, 104, 13-24. https://doi.org/10.1016/j.anbehav.2015.02.025

Samuni, L., Preis, A., Mielke, A., Deschner, T., Wittig, R. M., \& Crockford, C. (2018). Social bonds facilitate cooperative resource sharing in wild chimpanzees. Proceedings of the Royal Society B, 285(1888), 1-9. https://doi.org/10.1098/rspb.2018.1643

Sandel, A. A., Reddy, R. B., \& Mitani, J. C. (2017). Adolescent male chimpanzees do not form a dominance hierarchy with their peers. Primates, 58, 39-49. https://doi.org/10.1007/s10329-016-0553-z

Sapolsky, R. M., Alberts, S. C., \& Altmann, J. (1997). Hypercortisolism associated with social subordinance or social isolation among wild baboons. Archives of General Psychiatry, 54(12), 1137-1143. https:// doi.org/10.1001/archpsyc.1997.01830240097014

Schoof, V. A. M., \& Jack, K. M. (2014). Male social bonds: Strength and quality among co-resident white-faced capuchin monkeys (Cebus capucinus). Behaviour, 151(7), 963-992. https://doi.org/10.1163/ 1568539X-00003179

Schülke, O., Bhagavatula, J., Vigilant, L., \& Ostner, J. (2010). Social bonds enhance reproductive success in male macaques. Current Biology, 20(24), 2207-2210. https://doi.org/10.1016/j.cub.2010.10.058

Seyfarth, R., \& Cheney, D. (2012). The evolutionary origins of friendship. Annual Review of Psychology, 63, 153-157. https://doi.org/10.1146/ annurev-psych-120710-100337

Silk, J., Altmann, J., \& Alberts, S. (2006). Social relationships among adult female baboons (Papio cynocephalus) I. Variation in the strength of social bonds. Behavioral Ecology and Sociobiology, 61(2), 183-195. https://doi.org/10.1007/s00265-006-0249-2

Silk, J., Cheney, D., \& Seyfarth, R. (2013). A practical guide to the study of social relationships. Evolutionary Anthropology, 22(5), 213-225. https://doi.org/10.1002/evan.21367 
Silk, J. B. (2009). Nepotistic cooperation in non-human primate groups. Philosophical Transactions of the Royal Society B, 364(1533), 3243-3254. https://doi.org/10.1098/rstb.2009.0118.

Silk, J. B., Alberts, S. C., \& Altmann, J. (2003). Social bonds of female baboons enhance infant survival. Science, 302(5648), 1231-1234. https://doi.org/10.1126/science.1088580

Skaug, H., Fournier, D., Bolker, B., Magnusson, A., \& Nielsen, A. (2016). Generalized linear mixed models using 'AD model builder'. R package version 0.8.3.3. Retrieved from http://glmmadmb.r-forge.r-project.org

Smuts, B. (1985). Sex and friendship in baboons. New York, NY: Aldine Pub. Co.

Struhsaker, T. T. (1997). Ecology of an African rain forest: logging in Kibale and the conflict between conservation and exploitation. Gainesville, FL: University of Florida Press.

Thompson, N. A. (2019). Understanding the links between social ties and fitness over the life cycle in primates. Behaviour, 156(9), 859-908. https://doi.org/10.1163/1568539X-00003552.

Wakefield, M. L. (2008). Grouping patterns and competition among female Pan troglodytes schweinfurthii at Ngogo, Kibale National Park, Uganda. International Journal of Primatology, 29, 907-929. https://doi.org/10. 1007/s10764-008-9280-7

Watts, D., \& Mitani, J. (2001). Boundary patrols and intergroup encounters in wild chimpanzees. Behaviour, 138(3), 299-327. https://doi.org/10.1163/15685390152032488

Watts, D. P. (2000). Grooming between male chimpanzees at Ngogo, Kibale National Park. I. Partner number and diversity and grooming reciprocity. International Journal of Primatology, 21(2), 189-210. https://doi.org/10.1023/A:1005469302911

Watts, D. P. (2002). Reciprocity and interchange in the social relationships of wild male chimpanzees. Behaviour, 139(2/3), 343-370. https://doi. org/10.2307/4535926

Whitehead, H. (2008). Analyzing animal societies: Quantitative methods for vertebrate social analysis. Chicago, IL: The University of Chicago Press.

Wittig, R., Crockford, C., Lehmann, J., Whitten, P., Seyfarth, R., \& Cheney, D. (2008). Focused grooming networks and stress alleviation in wild female baboons. Hormones and Behavior, 54(1), 170-177. https://doi. org/10.1016/j.yhbeh.2008.02.009
Wittig, R. M., Crockford, C., Weltring, A., Langergraber, K. E., Deschner, T., \& Zuberbühler, K. (2016). Social support reduces stress hormone levels in wild chimpanzees across stressful events and everyday affiliations. Nature Communications, 7, 7. https://doi.org/10.1038/ncomms13361.

Wood, B. M., Watts, D. P., Mitani, J. C., \& Langergraber, K. E. (2017). Favorable ecological circumstances promote life expectancy in chimpanzees similar to that of human hunter-gatherers. Journal of Human Evolution, 105, 41-56. https://doi.org/10.1016/j.jhevol.2017. 01.003

Wroblewski, E., Murray, C., Keele, B., Schumacher-Stankey, J., Hahn, B., \& Pusey, A. (2009). Male dominance rank and reproductive success in chimpanzees, Pan troglodytes schweinfurthii. Animal Behaviour, 77(4), 873-885. https://doi.org/10.1016/j.anbehav.2008.12.014

Wroblewski, E. E. (2010). Paternity and father-offspring relationships in wild chimpanzees, Pan troglodytes schweinfurthii. (Unpublished doctoral dissertation), University of Minnesota, Minneapolis, MN.

Young, C., Majolo, B., Heistermann, M., Schülke, O., \& Ostner, J. (2014). Responses to social and environmental stress are attenuated by strong male bonds in wild macaques. Proceedings of the National Academy of Sciences, 111(51), 18195-18200. https://doi.org/10.1073/ pnas. 1411450111

\section{SUPPORTING INFORMATION}

Additional supporting information may be found online in the Supporting Information section.

How to cite this article: Sandel AA, Langergraber KE, Mitani JC. Adolescent male chimpanzees (Pan troglodytes) form social bonds with their brothers and others during the transition to adulthood. Am J Primatol. 2020;82:e23091.

https://doi.org/10.1002/ajp.23091 